\title{
Effects of illness duration and treatment resistance on grey matter abnormalities in major depression
}

Maria Serra-Blasco, Maria J. Portella, Beatriz Gómez-Ansón, Javier de Diego-Adeliño, Yolanda Vives-Gilabert, Dolors Puigdemont, Ester Granell, Alicia Santos, Enric Álvarez and Víctor Pérez

\section{Background}

Findings of brain structural changes in major depressive disorder are still inconsistent, partly because some crucial clinical variables have not been taken into account.

\section{Aims}

To investigate the effect of major depressive disorder on grey matter volumes.

\section{Method}

Voxel-based morphometry was used to compare 66 patients with depression at different illness stages (22 each with firstepisode, remitted-recurrent and treatment resistant/chronic depression) with 32 healthy controls. Brain volumes were correlated with clinical variables.

\section{Results}

Voxel-based morphometry showed a significant group effect in right superior frontal gyrus, left medial frontal gyrus and left cingulate gyrus $(P<0.05$, family wise error-corrected). Patients whose condition was treatment resistant/chronic exhibited the smallest volumes in frontotemporal areas.
Longer illness duration was negatively correlated with decreases in right medial frontal cortex and left insula.

\section{Conclusions}

Frontotemporolimbic areas are smaller in the patients with severe depression and are associated with duration of illness, but not with medication patterns, suggesting negative effects of long-lasting major depressive disorder on grey matter.

\section{Declaration of interest}

V.P. has received educational honoraria from: Sanofi-Aventis, Lundbeck, Pfizer, AstraZeneca and Eli Lilly, and research funding from Boehringer-Ingelheim for this work. E.A. has received consulting and educational honoraria from several pharmaceutical companies including Eli Lilly, Sanofi-Aventis, Lundbeck and Pfizer, and he has participated as main local investigator in clinical trials from Eli Lilly, Bristol-Myers Squibb and Sanofi-Aventis and also as national coordinator of clinical trials from Servier and Lundbeck.
One of the major concerns regarding major depressive disorder is that it shows the tendency to become chronic, ${ }^{1}$ with devastating consequences for patients such as a low quality of life, increased risk of mortality and elevated health and social costs. The pathophysiology of major depressive disorder at different stages of the illness is still unclear and the current neurobiological hypotheses exhibit some important weaknesses. ${ }^{2}$ Predominant neurobiological models are based on the occurrence of neurotoxic and neurotrophic processes before and during the disorder, including changes in grey matter volume that have been observed in brain structures of patients with major depressive disorder. ${ }^{3,4}$ Although the most replicated findings suggest losses of grey matter volume in frontolimbic areas, ${ }^{5}$ other neuroanatomical systems may be involved in major depressive disorder. Such diversity would better mirror the psychopathological heterogeneity of this disorder. A recent meta-analysis ${ }^{6}$ has reported that patients with remitted major depressive disorder have a significantly larger hippocampal volume compared with patients who are currently depressed. However, other clinical variables (e.g. number of previous episodes, illness onset) did not seem to be relevant in relation to grey matter volume. The different imaging techniques used in previous studies, the heterogeneity of samples and the limited overlap of results across imaging paradigms make it difficult to reliably identify neuronal regions or networks consistently affected in major depressive disorder. In addition, the fact that crucial clinical characteristics such as duration of illness have not been considered could partly explain some of the inconsistencies regarding the structures affected. For example, volumetric differences may be less marked in the early stages of the illness and more pronounced in advanced stages. We hypothesise that the clinical characteristics and the stage of the illness may affect the grey matter volume. The aims of this study were to investigate structural brain abnormalities at different stages of the illness and to determine the effect of clinical characteristics on brain grey matter volume.

\section{Method}

\section{Participants}

A total of 107 individuals were recruited for the present study, which is part of a bigger project investigating in vivo neuroimaging markers of clinical illness burden, ${ }^{7,8}$ and who underwent an magnetic resonance imaging (MRI) protocol specifically designed for this study. Nine patients had to be excluded from the study for technical or clinical reasons. The final sample included 66 individuals with major depressive disorder (DSM-IV-TR criteria) ${ }^{9}$ from the out-patients' psychiatric service of the Hospital Sant Pau in Barcelona, Spain, and 32 control individuals. All patients were on medication at the beginning of the study. Given that all patients were receiving different treatment regimens, a medication load index was calculated by taking the current drugs at the time of scanning following the system code proposed by Sackeim. ${ }^{10}$ The patients were split into three different groups. The first group ( $n=22$, treatment-resistant/chronic group) consisted of patients with a high burden of illness, with a diagnosis of chronic depressive disorder, a last episode duration of more than 2 years, no response to several antidepressant strategies, a Thase-Rush Index ${ }^{11}$ of treatment resistance $\geqslant 3$, and a score above 14 on the 
Hamilton Rating Scale for Depression (HRSD); ${ }^{12}$ the second group ( $n=22$, remitted-recurrent group) included patients who had experienced three or more previous episodes of major depressive disorder and were euthymic (HRSD $<8$ ) for the past 6 months. The third group ( $n=22$, first-episode group) comprised individuals with a first episode of major depressive disorder. Thirty-two healthy controls (control group) were also included. The exclusion criteria for healthy participants were: lifetime psychiatric diagnoses, first-degree relatives with psychiatric diagnoses and clinically significant physical or neurological illnesses. Semi-structured interviews were carried out for all participants to collect demographics and clinical information by two experienced psychiatrists. Axis I comorbidity according to DSM-IV-TR criteria was an exclusion criteria for all participants. Current depressive symptoms were assessed using the HRSD by experienced clinical researchers. All participating individuals were of a similar age (mean 46.86 years, s.d. $=7.99$ ) to avoid age-related variations in brain structures. The study was approved by the Research Ethics Committee of Hospital Sant Pau in Barcelona and was carried out in accordance with the Declaration of Helsinki. All participants gave informed and written consent after a full explanation of the study protocol.

\section{MRI data acquisition and processing procedures}

The MRIs were obtained using a 3T Philips Achieva facility (software version 2.1.3.2), three-dimensional (3D) shortest echo scans (repetition time $(\mathrm{TR})=6.7 \mathrm{~ms}$, echo time $(\mathrm{TE})=3.2 \mathrm{~ms}, 170$ slices, voxel size $(\mathrm{REC}): 0.89 \times 0.89 \times 1.2 \mathrm{~mm}$, image dimensions: $288 \times 288 \times 170$; field of view: $256 \times 256 \times 204 \mathrm{~mm}$, slice thickness: $1.2 \mathrm{~mm}$ ). For each participant, high-resolution 3D-MPRAGE images were acquired (whole brain coverage), with a sagittal slice orientation, $T_{1}$ contrast enhancement, flip angle: $8^{\circ}$, grey matter as a reference tissue, acquisition matrix $\mathrm{M} \times \mathrm{P}=256 \times 240$ and turbo-field echo shots $(\mathrm{TFE})=218$. All technical procedures were carried out in the cluster of the Port d'Informació Científica (PIC) on Scientific Linux 5 (www.scientificlinux.org/).

\section{VBM-DARTEL analysis}

The voxel-based morphometry (VBM) analyses were performed using SPM8 (www.fil.ion.ucl.ac.uk/spm) in a MATLAB 7.6.0 environment. First, MRIs were segmented into grey matter, white matter and cerebrospinal fluid using a standard segmentation model in SPM8. ${ }^{13}$ Second, grey matter templates were generated from the entire image data-set using the diffeomorphic anatomical registration and the exponentiated Lie algebra technique (DARTEL). ${ }^{14}$

Afterwards, an initial affine registration of the grey matter DARTEL templates to the tissue probability maps was carried out to create warped images. Images were then modulated to guarantee that grey matter relative volumes were preserved following the spatial normalisation procedure. Finally, images were smoothed with an $8 \mathrm{~mm}$ full-width at half maximum Gaussian kernel. Spatial pre-processing, smoothed, modulated, normalised grey matter data-sets were used to perform statistical analyses.

\section{Cortical volume}

Cortical surfaces were segmented using Freesurfer software for Linux (v.4.3.1, http://surfer.nmr.mgh.harvard.edu/) developed at the Martinos Center for Biomedical Imaging, to obtain the whole volume of every brain structure. Cortex volumes were obtained with the surface-based stream process, as described in detail in Fischl et $a l^{15}$ and Dale et al. ${ }^{16}$ First, the MRIs are affine registered to the Talairach atlas ${ }^{17}$ and image intensity variations as a result of magnetic field inhomogeneities are normalised. Then, a skull stripping algorithm is applied ${ }^{18}$ and the skull-stripped image is segmented into white and grey matter. Finally, the hemispheres are separated and the different surfaces are generated (white and grey). The distance between these surfaces gives the thickness at each location of the cortex. ${ }^{19}$ Following generation of cortical models, surface inflation and the register to a spherical atlas, a parcellation of the cerebral cortex into parts based on gyrus and sulcus structure are executed. ${ }^{20}$ The results of the cortical surface were verified by experts, and in some cases, manual modifications were applied to obtain more accurate results.

\section{Total intracranial volume measures}

Total intracranial volume was calculated in order to ensure that volume differences between participants were as a result of diagnosis instead of brain sizes. Given that two software tools were used, total intracranial volume was computed with both. To get the total intracranial volume provided by SPM8 $\left(\mathrm{TIV}_{\mathrm{spm}}\right)$, the spm_get_volumes function was used, and segmented grey, white and cerebrospinal fluid of each $\mathrm{rc}^{\star}$ (registered and segmented) image was then summed up. In the case of the total intracranial volume provided by Freesurfer $\left(\mathrm{TIV}_{\text {FreeSurfer }}\right)$, values given by automatic segmentation of volume-based stream were used.

\section{Data analyses}

Demographics and clinical variables were analysed using the $\mathrm{R}$ statistical package version 2.10.1 for Windows. ${ }^{21}$ Voxel-based morphometry was calculated using the DARTEL algorithm in SPM8 to quantify structural brain volumes. Group differences in absolute grey matter volume were assessed using ANOVA with subsequent post hoc comparisons. Absolute threshold mask was set at 0.2 , as recommended by John Ashburner in an VBM Tutorial, ${ }^{22}$ and other parameters were left at their default values. An additional ANCOVA with the three groups (first-episode, remitted-recurrent and treatment resistant/chronic) was performed to control for the effect of medication load (included as a covariate). Significant effects were considered using a $P<0.05$, corrected for multiple comparisons with family-wise error (FWE) for both omnibus (no extent threshold) and post hoc (cluster extent threshold $>100$ ) whole-brain tests.

Since SPM8 does not provide absolute volumes of a given brain region, FreeSurfer brain segmentation was used to obtain the corresponding volumes of those areas that showed significant group effects (cluster level $P$-value set at $<0.01$ ). These values were then correlated with relevant clinical variables such as HRSD scores, duration of illness, age at onset, medication load and number of previous episodes. Given the number of comparisons, significance level for correlation analyses was set at $P=0.01$. In order to determine the percentage of volume decrease attributable to clinical variables, an additional linear regression was performed where $x$ corresponded to clinical data and $y$ corresponded to volumes of brain structures. The resulting $y$ values were then divided by the interception of the regression model to get normalised values.

\section{Results}

\section{Participants}

A total of 98 participants entered the study. Table 1 shows the demographic, clinical and treatment data of patients and healthy controls. No significant differences between groups were observed in the demographic characteristics. Differences in HRSD scores, age at onset, medication load and duration of illness were as a 
result of patients classification based on the stage of the illness and the inclusion criteria. The first-episode group had a significantly older age at onset than the remitted-recurrent or treatmentresistant/chronic group $(F=20.9$, d.f. $=2,62, P<0.0001)$. However, this was a result of the age selection performed to minimise brain volume differences attributable to age. As expected, psychopharmacological treatments were unequally distributed across patient groups $(F=10.2$, d.f. $=2,63, P<0.0001)$. The treatmentresistant/chronic group were heavily treated, and frequently received concomitant treatment with other antidepressants, antipsychotics and/or stabilisers. There were no differences between groups with reference to $\operatorname{TIV}_{\text {spm }}(F=1.19$, d.f. $=3,94$, $P=0.32)$ or $\operatorname{TIV}_{\text {FreeSurfer }}(F=1.65$, d.f. $=3,94, P=0.18)$.

\section{VBM-DARTEL analyses (SPM8)}

The ANOVA of the control, first-episode, remitted-recurrent and treatment-resistant/chronic groups showed a significant group effect in right superior frontal gyrus (Brodmann area, BA 8), left medial frontal gyrus (BA 6) and left cingulate gyrus (BA 24) ( $F=11.10$, d.f. $=3,94, P_{\mathrm{FWE}}<0.05$, no extent threshold; Table 2). Post hoc contrast of the treatment-resistant/chronic group showed diminished grey matter volume compared with the control group $\left(t=4.75\right.$, d.f. $=1,94, P_{\mathrm{FWE}}<0.05$, extent threshold $k>100$ voxels), in right superior frontal gyrus (BA 8/9), left cingulate gyrus (BA 24 ), bilateral medial frontal gyrus (BA $6 / 8$ in left side and BA
10 in right side), left insula (BA 13), left inferior frontal gyrus (BA 44), left parahippocampal gyrus (BA 35), left transversetemporal gyrus (BA 21) and left post-central gyrus (BA 40). Results are detailed in Table 3. No other reductions or increments survived FWE corrections. Online Fig. DS1 represents the grey matter volume decreases in the treatment-resistant/chronic group compared with the control group.

There was a tendency of volume decrease in the remitted group compared with the control group $(t=3.87$, d.f. $=1,94$, $P<0.0001$ (uncorrected)) in right superior frontal gyrus (BA 8), right anterior lobe of cerebellum (culmen) and left cingulate gyrus (BA 24). Similarly, the treatment-resistant/chronic group also displayed a decrease of grey matter volume in comparison with the first-episode group $(t=3.87$, d.f. $=1,94, P<0.0001$ (uncorrected)) in left pre-central gyrus (BA 4), left post-central gyrus (BA 40), left medial frontal gyrus (BA 6), right insula (BA 13), right transversetemporal gyrus (BA 41), right inferior parietal lobule and left posterior cingulate (BA 30/31). Results are shown in Table 4.

\section{Effects of medication}

Mean values of the medication load index for each patient group are listed in Table 1 . The ANOVA of the three groups with depression (first episode, remitted-recurrent and treatmentresistant/chronic) did not show significant differences between groups $\left(F=15.12\right.$, d.f. $\left.=2,62, P>0.05, P_{\mathrm{FWE}}\right)$. The ANCOVA

\begin{tabular}{|c|c|c|c|c|c|c|}
\hline & $\begin{array}{l}\text { Healthy } \\
\text { control } \\
\text { group } \\
(n=32)\end{array}$ & $\begin{array}{l}\text { First-episode } \\
\text { group } \\
(n=22)\end{array}$ & $\begin{array}{l}\text { Remitted- } \\
\text { recurrent } \\
\text { group } \\
(n=22)\end{array}$ & $\begin{array}{l}\text { Treatment- } \\
\text { resistant/ } \\
\text { chronic group } \\
\quad(n=22)\end{array}$ & $F / \chi^{2}$ & $P$ \\
\hline \multicolumn{7}{|l|}{ Characteristics } \\
\hline Age, years: mean (s.d.) & $46(8.3)$ & $44(6.5)$ & $48(8.7)$ & $49(8)$ & 1.81 & 0.1501 \\
\hline Gender, $n$ & & & & & 4.19 & 0.24 \\
\hline Male & 9 & 7 & 2 & 4 & & \\
\hline Female & 23 & 15 & 20 & 18 & & \\
\hline Education, $n$ & & & & & 4.92 & 0.55 \\
\hline Primary school & 3 & 3 & 4 & 6 & & \\
\hline High school & 9 & 8 & 6 & 4 & & \\
\hline University & 20 & 11 & 9 & 11 & & \\
\hline Hamilton Rating Scale for Depression, ${ }^{a, c, d, e}$ mean (s.d.) & $2(1.7)$ & $16(6.5)$ & $4(5.2)$ & $21(4.6)$ & 94.12 & $<0.0001$ \\
\hline Age at onset, years: ${ }^{a, b}$ mean (s.d.) & NA & $43.5(6.6)$ & $29.7(11)$ & $27.4(8.4)$ & 20.90 & $<0.0001$ \\
\hline Time evolution, ${ }^{a, b}$ mean (s.d.) & NA & $5.6(4.2)$ & $214.3(129)$ & $271.5(145)$ & 38.57 & $<0.0001$ \\
\hline Episodes, $n:{ }^{a, b}$ mean (s.d.) & NA & $1(0)$ & $4.56(4.2)$ & $6.2(6.5)$ & 7.98 & 0.0008 \\
\hline \multicolumn{7}{|l|}{ Total intracranial volume, ml: mean (s.d.) } \\
\hline SPM8 & $16176(2563)$ & $16701(1892)$ & $17233(1783)$ & 16402 (1783) & 1.19 & 0.32 \\
\hline FreeSurfer & $11685(1699)$ & $12357(2301)$ & $11652(1562)$ & $11143(1685)$ & 1.65 & 0.18 \\
\hline \multicolumn{7}{|l|}{ Treatment } \\
\hline \multicolumn{7}{|l|}{ Antidepressants, $^{f} n(\%)$} \\
\hline $\begin{array}{l}\text { Selective serotonin reuptake inhibitors or selective } \\
\text { serotonin-noradrenaline reuptake inhibitors }\end{array}$ & - & $20(100)$ & $15(75)$ & $19(86)$ & 5.27 & 0.3 \\
\hline Tricyclic antidepressants or monoamine oxidase inhibitors ${ }^{a, b}$ & - & $0(0)$ & $3(15)$ & $8(36)$ & 11.97 & 0.018 \\
\hline Others $s^{b, d}$ & - & $1(0.5)$ & $1(0.5)$ & $12(57)$ & 13.13 & 0.011 \\
\hline Combination ${ }^{a, b, d}$ & - & $2(11)$ & $4(20)$ & $17(77)$ & 26.84 & $<0.0001$ \\
\hline No antidepressant & - & $0(0)$ & $2(10)$ & $0(0)$ & 6.4 & 0.17 \\
\hline Stabilisers, ${ }^{g}$ (\%) & - & $3(16)$ & $4(20)$ & $8(36)$ & 5.09 & 0.28 \\
\hline Antipsychotics, b, d,h $n$ (\%) & - & $2(11)$ & $2(10)$ & $10(45)$ & 12.64 & 0.013 \\
\hline Benzodiazepine, n (\%) & - & $5(26)$ & $6(30)$ & $13(59)$ & 8.38 & 0.08 \\
\hline \multicolumn{7}{|c|}{$\begin{array}{l}\text { NA, not applicable. } \\
\text { a. Significant differences between the first-episode and remitted-recurrent group. } \\
\text { b. Significant differences between the first-episode and treatment-resistant/chronic group. } \\
\text { c. Significant differences between the first-episode and control group. } \\
\text { d. Significant differences between the treatment-resistant/chronic and remitted-recurrent group. } \\
\text { e. Significant differences between the treatment-resistant/chronic and control group. } \\
\text { f. Antidepressants. Others: noradrenaline reuptake inhibitors, noradrenaline and dopamine reuptake inhibitors, tetracyclic antidepressants, mirtazapine, methylphenidate or trazodone } \\
\text { Combination: concomitant use of antidepressants with different mechanisms of action (for example selective serotonin reuptake inhibitors with reboxetine). } \\
\text { g. Anticonvulsants and mostly lithium. } \\
\text { h. Mainly atypical antipsychotics associated with antidepressants. }\end{array}$} \\
\hline
\end{tabular}




\begin{tabular}{|c|c|c|c|c|c|c|c|}
\hline \multirow[b]{2}{*}{ Anatomical region } & \multicolumn{2}{|c|}{ Test value } & \multirow[b]{2}{*}{ Cluster size } & \multirow[b]{2}{*}{ Cluster level } & \multicolumn{3}{|c|}{ MNI coordinates ${ }^{b}$} \\
\hline & $F$ & $z$ & & & $x$ & $y$ & $z$ \\
\hline Right superior frontal gyrus (BA 8) & 14.63 & 5.27 & 179 & 0.005 & 4 & 33 & 49 \\
\hline Left cingulate gyrus (BA 24) & 12.66 & 4.89 & 30 & 0.009 & -14 & 6 & 36 \\
\hline Left medial frontal gyrus (BA 6) & 12.35 & 4.83 & 37 & 0.016 & -10 & -5 & 65 \\
\hline
\end{tabular}

\begin{tabular}{|c|c|c|c|c|c|c|c|}
\hline \multirow[b]{2}{*}{ Contrast/regions } & \multicolumn{2}{|c|}{ Test value } & \multirow[b]{2}{*}{ Cluster size } & \multirow[b]{2}{*}{ Peak level } & \multicolumn{3}{|c|}{ MNI coordinates ${ }^{b}$} \\
\hline & $F$ & $Z$ & & & $x$ & $y$ & $z$ \\
\hline Control group > first-episode group & - & - & - & - & - & - & - \\
\hline Control group > remitted-recurrent group & - & - & - & - & - & - & - \\
\hline \multicolumn{8}{|l|}{ Control group > treatment-resistant/chronic group } \\
\hline Right superior frontal gyrus (BA 8) & 6.15 & 5.62 & 877 & $<0.001$ & 5 & 34 & 49 \\
\hline Right superior frontal gyrus (BA 9) & 5.33 & 4.97 & & & 5 & 51 & 36 \\
\hline Right medial frontal gyrus (BA 10) & 5.31 & 4.96 & & & 2 & 60 & 17 \\
\hline Left cingulate gyrus (BA 24) & 5.88 & 5.41 & 641 & 0.001 & -14 & 7 & 36 \\
\hline Left cingulate gyrus (BA 24) & 4.97 & 4.67 & & & -6 & -3 & 34 \\
\hline Left medial frontal gyrus (BA 6) & 5.78 & 5.33 & 123 & 0.006 & -11 & -5 & 65 \\
\hline Left insula (BA 13) & 5.69 & 5.26 & 767 & 0.002 & -48 & 12 & -0 \\
\hline Left inferior frontal gyrus (BA 44) & 5.38 & 5.01 & & & -56 & 9 & 13 \\
\hline Left medial frontal gyrus (BA 8) & 5.30 & 4.94 & 192 & 0.011 & -9 & 38 & 46 \\
\hline Left parahippocampal gyrus (BA 35) & 5.06 & 4.75 & 180 & 0.022 & -24 & -10 & -32 \\
\hline Left transverse-temporal gyrus (BA 21) & 4.99 & 4.69 & 114 & 0.019 & -59 & -20 & 14 \\
\hline Left post-central gyrus (BA 40) & 4.85 & 4.57 & & & -61 & -29 & 18 \\
\hline First-episode group > remitted-recurrent group & - & - & - & - & - & - & - \\
\hline First-episode group > treatment-resistant/chronic group & - & - & - & - & - & - & - \\
\hline \multicolumn{8}{|l|}{ Remitted-recurrent group > treatment-resistant/chronic group } \\
\hline Control group $<$ first-episode group & - & - & - & - & - & - & - \\
\hline Control group < remitted-recurrent group & - & - & - & - & - & - & - \\
\hline Control group $<$ treatment-resistant/chronic group & - & - & - & - & - & - & - \\
\hline First-episode group < remitted-recurrent group & - & - & - & - & - & - & - \\
\hline First-episode group < treatment-resistant/chronic group & - & - & - & - & - & - & - \\
\hline Remitted-recurrent group < treatment-resistant/chronic group & - & - & - & - & - & - & - \\
\hline \multicolumn{8}{|c|}{$\begin{array}{l}\text { FWE-Corr, corrected for multiple comparisons with family-wise error. } \\
\text { a. Anatomical region based on Talairach Atlas, } t \text { and } Z \text { scores, spatial extent in number of voxels (cluster size) and Montreal Neurological Institute (MNI) coordinates of the most } \\
\text { significant voxel of each cluster are displayed. Extent threshold: } 100 \text {. } \\
\text { b. The coordinates within each cluster were converted from MNI spatial array to the stereotaxic array of Talairach and Tournoux }{ }^{16} \text { using a non-linear transformation. }\end{array}$} \\
\hline
\end{tabular}

including the medication load as the covariate also failed to detect significant group effects $\left(F=15.04\right.$; d.f. $\left.=2,62, P>0.05, P_{\mathrm{FWE}}\right)$.

\section{Correlations between segmented brain volumes (Freesurfer) and clinical characteristics}

Table 5 displays absolute volumes of the segmented regions in $\mathrm{ml}$ (left anterior cingulate, right superior frontal gyrus, bilateral medial frontal gyrus and left insula). Group effects were only observed in right and left medial frontal gyri $(F=4.2$, d.f. $=3,94$, $P=0.008$ and $F=3.52$, d.f. $=3,94, P=0.018$ respectively) and left insula $(F=3.19$, d.f. $=3,94, P=0.027)$. In post hoc analyses, individuals in the treatment-resistant/chronic group had less grey matter volume than those in the first-episode group in right medial frontal gyrus $(P=0.011)$ and left insula $(P=0.03)$. In addition, the chronic group also showed less volume than the remitted-recurrent group in both sides of medial frontal gyrus (right: $P=0.02$, left: $P=0.01$ ). Correlation analyses showed that duration of illness was significantly correlated with right medial frontal cortex $(r=-0.34, P=0.006)$ and with left insula $(r=-0.3, P=0.01$; online Fig. DS2). Linear regression analysis predicted $19 \%$ of grey matter volume reductions in right medial frontal gyrus and $11.4 \%$ in left insula. The rest of the clinical variables did not correlate with those areas showing significant volume reductions.

\section{Discussion}

\section{Main results}

The findings of the present study suggest that highly deleterious structural brain changes occur in patients exhibiting a more severe and chronic depressive disorder. Grey matter volume reductions in frontolimbic areas were observed in patients with long-lasting illness and with no response to treatment strategies, providing evidence of the implication of this neural circuitry in the changing pathophysiology of major depressive disorder. The observed 


\begin{tabular}{|c|c|c|c|c|c|c|c|}
\hline \multirow[b]{2}{*}{ Contrast/regions } & \multicolumn{2}{|c|}{ Test value } & \multirow[b]{2}{*}{ Cluster size } & \multirow[b]{2}{*}{ Peak level } & \multicolumn{3}{|c|}{ MNI coordinates ${ }^{b}$} \\
\hline & $F$ & z & & & $x$ & y & $z$ \\
\hline Control group > first-episode group & - & - & - & - & - & - & - \\
\hline \multicolumn{8}{|l|}{ Control group > remitted-recurrent group } \\
\hline Right superior frontal gyrus (BA 8) & 4.88 & 4.6 & 239 & $<0.0001$ & 4 & 31 & 51 \\
\hline Right cerebellum (culmen) & 4.46 & 4.24 & 415 & $<0.0001$ & 43 & -40 & -34 \\
\hline Left cingulate gyrus (BA 24) & 4.04 & 3.87 & 164 & $<0.0001$ & -13 & -26 & 31 \\
\hline \multicolumn{8}{|l|}{ Control group > treatment-resistant/chronic group ${ }^{c}$} \\
\hline First-episode group $>$ remitted-recurrent group & - & - & - & - & - & - & - \\
\hline \multicolumn{8}{|l|}{ First-episode group > treatment-resistant/chronic group } \\
\hline Left pre-central gyrus (BA 4) & 4.9 & 4.61 & 890 & $<0.0001$ & -58 & -14 & 32 \\
\hline Left medial frontal gyrus (BA 6) & 4.69 & 4.43 & 116 & $<0.0001$ & -7 & -7 & 64 \\
\hline Right insula (BA 13) & 4.55 & 4.31 & 248 & $<0.0001$ & 48 & -16 & 3 \\
\hline Right transverse temporal gyrus (BA 41) & 4.5 & 4.28 & 216 & $<0.0001$ & 61 & -19 & 12 \\
\hline Right inferior parietal lobule (BA 40) & 4.44 & 4.22 & 109 & $<0.0001$ & 59 & -45 & 21 \\
\hline Left posterior cingulate (BA 30) & 4.29 & 4.09 & 120 & $<0.0001$ & -10 & -70 & 10 \\
\hline Left posterior cingulate (BA 31) & 4.27 & 4.09 & 153 & $<0.0001$ & -8 & -55 & 24 \\
\hline \multicolumn{8}{|l|}{ Remitted-recurrent group > treatment-resistant/chronic group } \\
\hline \multicolumn{8}{|l|}{ Control group $<$ first-episode group } \\
\hline Control group < remitted-recurrent group & - & - & - & - & - & - & - \\
\hline \multicolumn{8}{|l|}{ Control group $<$ treatment-resistant/chronic group } \\
\hline First-episode group < remitted-recurrent group & - & - & - & - & - & - & - \\
\hline First-episode group < treatment-resistant/chronic group & - & - & - & - & - & - & - \\
\hline Remitted-recurrent group < treatment-resistant/chronic group & - & - & - & - & - & - & - \\
\hline $\begin{array}{l}\text { a. Anatomical region based on Talairach Atlas, } t \text { and } Z \text { scores, spatial ext } \\
\text { significant voxel of each cluster are displayed. Extent threshold: } 100 \text {. } \\
\text { b. The coordinates within each cluster were converted from MNI spatial } \\
\text { c. Results not reported: this contrast was not run given that it was alread }\end{array}$ & the & Iuster & $\begin{array}{l}\text { and Montreal } \\
\text { ach and Tourn }\end{array}$ & $\begin{array}{l}\text { urological Ins } \\
x^{16} \text { using a n }\end{array}$ & (MNI) & nates of & \\
\hline
\end{tabular}

\begin{tabular}{|c|c|c|c|c|}
\hline \multirow[b]{2}{*}{ Brain region } & \multicolumn{4}{|c|}{ Mean (s.d.) } \\
\hline & $\begin{array}{l}\text { Healthy control group } \\
\qquad(n=32)\end{array}$ & $\begin{array}{l}\text { First-episode group } \\
\qquad(n=22)\end{array}$ & $\begin{array}{l}\text { Remitted-recurrent group } \\
\qquad(n=22)\end{array}$ & $\begin{array}{l}\text { Treatment-resistant/chronic group } \\
\qquad(n=22)\end{array}$ \\
\hline Left anterior cingulate & $1588(380)$ & $1638(350)$ & $1733(452)$ & $1536(403)$ \\
\hline Right superior frontal gyrus & 18097 (2381) & 17988 (1868) & $17927(2756)$ & $16689(2347)$ \\
\hline Right medial frontal gyrus & 3969 (395) & 4198 (679) & 4160 (677) & 3646 (585) \\
\hline Left medial frontal gyrus & 3628 (384) & $3761(687)$ & 3891 (581) & 3408 (412) \\
\hline Left insula & $5462(526)$ & $5857(563)$ & $5676(685)$ & 5365 (615) \\
\hline
\end{tabular}

differences were clearer when considering clinical variables related to the severity of the disorder. These findings suggest that grey matter abnormalities are directly correlated with past illness burden. The secondary analyses (using FreeSurfer) showed that individuals in the treatment-resistant/chronic group had smaller volumes in the segmented right medial frontal gyrus and left insula in comparison with those in the first-episode group, a result that was supported by the negative correlation between these two areas and duration of illness. This finding supports the potential risk of a history of severe illness on brain structures and the apparent brain preservation in the first stages of the illness. Moreover, the remitted-recurrent group showed bigger bilateral medial frontal gyrus volumes than the treatment-resistant/chronic group. This observation suggests a specific involvement of this area in maintaining depressive symptoms and refractoriness, and it is one of the targets for deep brain stimulation in patients with depression that is treatment resistant. ${ }^{23}$

Previous studies reported that clinical outcome (response to antidepressant treatments) had a direct effect on grey matter volume in the prefrontal cortex of patients. ${ }^{24}$ Duration of illness has also been related to greater grey matter reductions. ${ }^{4}$ However, little attention has been paid to factors related to treatment nonresponse, whether this was as a result of a lack of response to the treatment strategy or whether patients experienced a more severe form of treatment resistance. Our findings revealed that only those patients with treatment-resistant/chronic major depressive disorder showed differences related to other clinical characteristics such as duration of illness, age at onset or number of previous episodes rather than to current symptomatology or medication load. The brain areas that seem to bear the deleterious effects of depression mainly coincide with those previously reported in patients whose condition was non-remitting: dorsolateral-prefrontal cortex, cingulate cortex, hippocampus, and medial prefrontal cortex. ${ }^{24}$ In addition, a 7 -year follow-up study ${ }^{25}$ reported that patients with slower recovery exhibited decreased volumes of left insula, hippocampus and lateral parietal cortex. Therefore, less grey matter volume in superior and medial prefrontal cortex, cingulate gyrus, insula and parahippocampal gyrus seem to be responsible for the persistence of depressive symptoms, hampering illness recovery.

In spite of the previous findings, the aetiology of brain volume decrease remains unclear. A review by Drevets ${ }^{26}$ identified 
elevations of glutamate transmission and cortisol hypersecretion in major depressive disorder and suggested that grey matter volume reductions in participants with current depression could be partly explained by interactions between elevated glucocorticoid secretion and $\mathrm{N}$-methyl-D-aspartate (NMDA)-glutamate receptor stimulation. Gold $e t a l^{27}$ also reported that the protective/ neurotrophic effects exerted by some antidepressant drugs may prevent and restore the volumetric alterations. However, an inadequate response to antidepressant strategies would most likely preclude these improvements and may even lead to a worsening as a consequence of sustained stress. These findings support the neurotoxic hypothesis, whereby a brain volume loss exists during the course of depressive illness, caused by glucocorticoid and glutamatergic toxicity, and a decrease in neurotrophic factors and neurogenesis. ${ }^{25}$

These possible neurotoxic effects cannot be investigated in our sample for two reasons: first, although the analyses took into account the effects of medication load it is not possible to know whether patients became resistant because of previous small grey matter volume or because of the toxicity associated with longterm medication. Second, the treatment-resistant/chronic group had not been followed up from the beginning of the illness. The participants with treatment-resistant/chronic disorder had been on long-term pharmacotherapy and had received more treatment combinations (as determined by medication load index) than the other groups of patients included. In any case, the impact of being exposed to antidepressant drugs would have not been beneficial and may have entailed greater impairment on the brain areas investigated. Unfortunately, there are few studies with drug-naive major depressive disorder samples. A recent study reported thinner cortical thickness in patients with depression with a late onset who were drug-naive compared with healthy controls. ${ }^{28}$ The affected areas were located in frontotemporal and posterior cingulate cortex. Previous studies on patients who were drugnaive showed inconsistent results about which areas show decreases in grey matter volume, and many of these studies have been reported with uncorrected significance values. It is possible that in the case of treatment resistance, both factors, being depressed for a long period of time and not responding to antidepressant combinations, contribute to the apparent brain damage. Further studies are needed to clarify the effects of medication on grey matter volumes.

\section{Limitations of the study}

This is a cross-sectional study and therefore the harmful effects of depression on grey matter volume could not be evaluated. Nevertheless, two different types of post-processing software were used to test our hypothesis about the impact of illness burden on brain structures. Both found similar differences within the medial frontal gyrus confirming our hypothesis. In addition, all main results were strictly corrected for multiple comparisons. The present study may also be limited by the older age at onset of patients with a first episode, which might cast doubt about the representativeness of this sample. Although there was no significant relationship between age at onset and brain volumes, a later onset has been associated with a better prognosis in major depressive disorder. ${ }^{29}$ Nonetheless, this sample of individuals in the first-episode group is similar in age to the other investigated patients, providing a good comparison group to control for illness burden, and minimising the confounding effects of age-related changes in brain structures. Additionally, the grouping of patients performed in this study offers the possibility to compare patients with depression at different and well-defined stages of illness. Finally, our findings may be limited by the lack of a treatment washout period, although withdrawing antidepressant treatment to severely ill patients would constitute an ethical issue. Moreover, treatment regimens differed among groups: the treatmentresistant/chronic group, in particular, received combined treatments more frequently. Treatment effects on grey matter have not been well established yet but some evidence have suggested that antidepressant drugs may even attenuate volume decreases after successful treatment and remission. ${ }^{24,30}$ Nevertheless, we included an index of medication load in VBM ANCOVA with no changes in the results.

In conclusion, frontolimbic areas were reduced in the individuals who were the most severely depressed, namely those in the treatment-resistant/chronic group. The insula and the medial frontal gyrus are the most affected brain regions, which may underlie the varying pathophysiology of major depressive disorder. Further research is needed to investigate the preservation of these brain structures, known to play key roles in regulating endocrine, autonomic, behavioural and emotional responses.

Maria Serra-Blasco, BSC, Department of Psychiatry, Hospital de la Santa Creu i Sant Pau, Universitat Autònoma de Barcelona (UAB), Institut d'Investigació Biomèdica Sant Pau (IIB Sant Pau), Centro de Investigación Biomédica en Red de Salud Mental (CIBERSAM) and Port d'Informació Científica (PIC), Universitat Autònoma de Barcelona (UAB), Barcelona; Maria J. Portella, PhD, Department of Psychiatry, Hospital de la Santa Creu i Sant Pau, Universitat Autonoma de Barcelona (UAB), Institut d'Investigació Biomèdica Sant Pau (IIB Sant Pau), Centro de Investigación Biomédica en Red de Salud Mental (CIBERSAM), Barcelona; Beatriz Gómez-Ansón, MD, PhD, Department of Neuroradiology, Hospital de la Santa Creu i Sant Pau, Universitat Autònoma de Barcelona (UAB), Institut d'Investigació Biomèdica Sant Pau (IIB Sant Pau), Centro de Investigación Biomédica en Red para Enfermedades Neurodegenerativas (CIBERNED), Barcelona; Javier de Diego-Adeliño, MD, MHS, Department of Psychiatry, Hospital de la Santa Creu i Sant Pau, Universitat Autònoma de Barcelona (UAB), Institut d'Investigació Biomèdica Sant Pau (IIB Sant Pau), Centro de Investigación Biomédica en Red de Salud Mental (CIBERSAM), Barcelona; Yolanda Vives-Gilabert, PhD, Port d'IInformació Científica (PIC), Universitat Autònoma de Barcelona (UAB), Barcelona; Dolors Puigdemont, MD, Department of Psychiatry, Hospital de la Santa Creu i Sant Pau, Universitat Autònoma de Barcelona (UAB), Institut d'Investigació Biomèdica Sant Pau (IIB Sant Pau), Centro de Investigación Biomédica en Red de Salud Mental (CIBERSAM), Barcelona; Ester Granell, MD, Biomédica en Red de Salud Mental (CIBERSAM), Barcelona; Ester Granell, MD,
Department of Neuroradiology, Hospital de la Santa Creu i Sant Pau, Universitat Autònoma de Barcelona (UAB), Institut d'Investigació Biomèdica Sant Pau (IIB Sant Pau), Centro de Investigación Biomédica en Red para Enfermedades Neurodegenerativas (CIBERNED), Barcelona; Alicia Santos, BSC, Department of Endocrinology, Hospital de la Santa Creu i Sant Pau, Universitat Autònoma de Barcelona (UAB), Institut d'Investigació Biomèdica Sant Pau (IIB Sant Pau), Centro de Investigación Biomédica en Red de Enfermedades Raras (CIBERER), Barcelona; Enric Álvarez, MD, PhD, Víctor Pérez, MD, PhD, Department of Psychiatry, Hospital de la Santa Creu i Sant Pau, Universitat Autònoma de Barcelona (UAB), Institut d'Investigació Biomèdica Sant Pau (IIB Sant Pau), Centro de Investigación Biomédica en Red de Salud Mental (CIBERSAM), Barcelona, Spain

Correspondence: Maria J. Portella, Department of Psychiatry, Hospital de la Santa Creu i Sant Pau, Institut d'Investigacions Biomèdiques de Sant Pau (IIB-Sant Pau); Centro de Investigación Biomedica en Red de Salud Mental (CIBERSAM), Sant Antoni Ma. Claret 167, 08025 Barcelona, Catalonia, Spain. Email: MPortella@santpau.cat

First received 11 Jun 2012, final revision 3 Dec 2012, accepted 18 Feb 2013

\section{Funding}

This study was funded by two grants: the Fondo de Investigación Sanitaria (FIS: PI 10/ 00372; FIS: 07/00770) from the Instituto de Salud Carlos III, by the Centro de Investigación Biomédica en Red de Salud Mental (CIBERSAM) and by the Centro de Investigación Biomédica para Enfermedades Neurodegenerativas (CIBERNED). M.S.-B. is funded by the Agència de Gestió d'Ajuts Universitaris i de Recerca of the Generalitat de Catalunya through a pre-doctorate fellowship (FI-DGR 2012). M.J.P. is funded by the Ministerio de Ciencia e Innovación of the Spanish Government and by the Instituto de Investigación Carlos III through a 'Miguel Servet' research contract (CP10-00393), co-financed by the European Regional Development Fund (ERDF) (2007-2013). J.de.D.-A. is funded by the Instituto de Salud Carlos III through a 'Rio Hortega' research fellowship.

\section{Acknowledgements}

We thank the staff of the medical imaging group at Port d'Informació Científica (PIC) and the Deparment of Psychiatry and Neuroradiology, Hospital de la Santa Creu i Sant Pau for their assistance in this study. We owe special gratitude to Dr Arranz for her thoughtful for their assistance in this study. We owe special gratitude to Dr Arranz for her thoughtful
comments on the revised version of the manuscript. We also thank the patients who participated in the current study for their kind cooperation. 


\section{References}

1 Rubio JM, Markowitz JC, Alegría A, Pérez-Fuentes G, Liu S-M, Lin K-H, et al. Epidemiology of chronic and nonchronic major depressive disorder: results from the national epidemiologic survey on alcohol and related conditions. Depress Anxiety 2011; 28: 622-31.

2 Hasler G. Pathophysiology of depression: do we have any solid evidence of interest to clinicians? World Psychiatry 2010; 9: 155-61.

3 Du M-Y, Wu Q-Z, Yue Q, Li J, Liao Y, Kuang W-H, et al. Voxelwise metaanalysis of gray matter reduction in major depressive disorder. Prog Neuropsychopharmacol Biol Psychiatry 2012; 10: 11-6.

4 Bora E, Fornito A, Pantelis C, Yücel M. Gray matter abnormalities in major depressive disorder: a meta-analysis of voxel based morphometry studies. J Affect Disord 2012; 138: 9-18.

5 Sacher J, Neumann J, Fünfstück T, Soliman A, Villringer A, Schroeter ML. Mapping the depressed brain: a meta-analysis of structural and functional alterations in major depressive disorder. J Affect Disord 2011; 140: 142-8.

6 Kempton MJ, Salvador Z, Munafò MR, Geddes JR, Simmons A, Frangou S, et al. Structural neuroimaging studies in major depressive disorder. Meta-analysis and comparison with bipolar disorder. Arch Gen Psychiatry 2011; 68: 675-90.

7 Portella MJ, de Diego-Adeliño J, Gómez-Ansón B, Morgan-Ferrando R, Vives $Y$, Puigdemont D, et al. Ventromedial prefrontal spectroscopic abnormalities over the course of depression: a comparison among first episode, remitted recurrent and chronic patients. J Psychiatr Res 2011; 45 427-34.

8 De Diego-Adeliño J, Portella MJ, Gómez-Ansón B, López-Moruelo O, Serra-Blasco M, Vives $Y$, et al. Hippocampal abnormalities of glutamate/ glutamine, $\mathrm{N}$-acetylaspartate and choline in patients with depression are related to past illness burden. J Psychiatry Neurosci 2012; Sept 4 (Epub ahead of print).

9 American Psychiatric Association. Diagnostic and Statistical Manual of Mental Disorders (4th edn, text revision) (DSM-IV-TR). APA, 2000.

10 Sackeim HA. The definition and meaning of treatment-resistant depression. J Clin Psychiatry 2001; 16: 10-7.

11 Thase ME, Rush AJ. When at first you don't succeed: sequential strategies for antidepressant nonresponders. J Clin Psychiatry 1997; 58: 23-9.

12 Hamilton M. A rating scale for depression. I Neurol Neurosurg Psychiatry 1960; 23: 56-62.

13 Ashburner J, Friston KJ. Unified segmentation. Neuroimage 2005; 26: 839-51.

14 Ashburner J. A fast diffeomorphic image registration algorithm. Neuroimage 2007; 15: 95-113.

15 Fischl B, Sereno MI, Tootell RB, Dale AM. High-resolution intersubject averaging and a coordinate system for the cortical surface. Hum Brain Mapp 1999; 8: 272-84.
16 Dale AM, Fischl B, Sereno MI. Cortical surface-based analysis. I. Segmentation and surface reconstruction. Neuroimage 1999; 9: 179-94.

17 Talairach J, Tournoux P. Co-Planar Stereotaxic Atlas of the Human Brain: An Approach to Medical Cerebral Imaging. Thieme Classics, 1988.

18 ségonne F, Dale AM, Busa E, Glessner M, Salat D, Hahn HK, et al. A hybrid approach to the skull stripping problem in MRI. Neuroimage 2004; 22: 1060-75.

19 Fischl B, Dale AM. Measuring the thickness of the human cerebral cortex from magnetic resonance images. Proc Natl Acad Sci USA 2000; 26: 11050-5.

20 Desikan RS, Ségonne F, Fischl B, Quinn BT, Dickerson BC, Blacker D, et al. An automated labeling system for subdividing the human cerebral cortex on MRI scans into gyral based regions of interest. Neuroimage 2006; 1: 968-80.

21 R Development Core Team. R: A Language and Environment for Statistical Computing. R Development Core Team, 2009 (http://www.R-project.org).

22 Ashburner J. VBM Tutorial. March 15 2010. UCL, 2010 (http:// www.fil.ion.ucl.ac.uk/ john/misc/VBMclass10.pdf).

23 Mayberg HS, Lozano AM, Voon V, McNeely HE, Seminowicz D, Hamani C, et al. Deep brain stimulation for treatment-resistant depression. Neuron 2005; 3: 651-60.

24 Salvadore G, Nugent AC, Lemaitre H, Luckenbaugh DA, Tinsley R, Cannon DM, et al. Prefrontal cortical abnormalities in currently depressed versus currently remitted patients with major depressive disorder. Neuroimage 2011; 14: 2643-51.

25 Soriano-Mas C, Hernández-Ribas R, Pujol J, Urretavizcaya M, Deus J, Harrison BJ, et al. Cross-sectional and longitudinal assessment of structural brain alterations in melancholic depression. Biol Psychiatry 2011; 15: 318-25.

26 Drevets WC. Neuroplasticity in mood disorders. Dialogues Clin Neurosci 2004; 6: 199-216.

27 Gold PW, Drevets WC, Charney DS. New insights into the role of cortisol and the glucocorticoid receptor in severe depression. Biol Psychiatry 2002; 1 : $381-5$.

28 Lim HK, Jung WS, Ahn KJ, Won WY, Hahn C, Lee SY, et al. Regional cortical thickness and subcortical volume changes are associated with cognitive impairments in the drug-naive patients with late-onset depression. Neuropsychopharmacology 2012; 37: 838-49.

29 Souery D, Oswald P, Massat I, Bailer U, Bollen J, Demyttenaere K, et al. Clinical factors associated with treatment resistance in major depressive disorder: results from a European multicenter study. J Clin Psychiatry 2007; 68: 1062-70.

30 Arnone D, McKie S, Elliott R, Juhasz G, Thomas EJ, Downey D, et al State-dependent changes in hippocampal grey matter in depression. Mol Psychiatry 2012; Nov 6 (Epub ahead of print). 